\title{
Estimation of optical parameters of silicon single crystals with different orientations
}

\author{
M.M. EL-NAHASS, H.A.M. Ali* \\ Department of Physics, Faculty of Education, Ain Shams University, Roxy 11757, Cairo, Egypt
}

\begin{abstract}
Optical properties of Si single crystals with different orientations (lllllll 000 and $\left(\begin{array}{lll}1 & 1 & 1\end{array}\right)$ were investigated using spectrophotometric measurements in a spectral range of $200 \mathrm{~nm}$ to $2500 \mathrm{~nm}$. The data of optical absorption revealed an indirect allowed transition with energy gap of $1.1 \pm 0.025 \mathrm{eV}$. An anomalous dispersion in addition to a normal dispersion was observed in the spectra of refractive index. The normal dispersion of the refractive index was discussed according to Wemple-DiDomenico single oscillator model. The oscillator energy $\mathrm{E}_{\mathrm{o}}$, dispersion energy $\mathrm{E}_{\mathrm{d}}$, high frequency dielectric constant $\epsilon_{\infty}$, lattice dielectric constant $\epsilon_{L}$ and electronic polarizability $\alpha_{\mathrm{e}}$ were estimated. The real $\epsilon_{1}$ and imaginary $\epsilon_{2}$ parts of dielectric constant were also determined.
\end{abstract}

Keywords: single crystals; different orientations; optical parameters

\section{Introduction}

Silicon is a commonly used semiconductor in optoelectronic devices and photodiodes, which are broadly used in industrial applications as effective devices for light to electricity conversion [1]. The availability of high-quality single crystal and welldeveloped processing technologies stimulate new directions in investigations of the properties of silicon [2]. The field of research associated with optical properties of semiconductors has seen an enormous growth over the last decade [3]. The knowledge of optical material properties is essential in the design of electro-optical devices, such as solar cells. The primary aim of both theoretical and experimental investigations of silicon crystals is to elucidate the origin of visible light emission [4].

This work aims to investigate the optical dispersion parameters of silicon single crystals with different orientations on the basis of dispersion and polarization theories.

*E-mail: hend2061@yahoo.com

\section{Experimental}

The p-silicon single crystals used in the experiments were supplied from Spectra Chemical Industries. The samples of dimensions $6 \mathrm{~mm} \times 4 \mathrm{~mm} \times 0.45 \mathrm{~mm}$ and a resistivity of

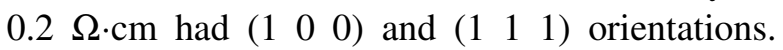
They were highly polished to a mirror-like surface. The transmission $\mathrm{T}(\lambda)$, at normal incidence, as well as the reflection $\mathrm{R}(\lambda)$, at an incident angle of $5^{\circ}$, were measured in the spectral range of $\lambda$ from $200 \mathrm{~nm}$ to $2500 \mathrm{~nm}$ using a double beam spectrophotometer (JASCO, V-570 UV-Vis-NIR). The measurements were done for Si crystals with two different orientations ( $\left(\begin{array}{lll}1 & 0 & 0\end{array}\right)$ and $\left(\begin{array}{lll}1 & 1 & 1\end{array}\right)$. The experimental error for measured $\mathrm{T}$ and $\mathrm{R}$ was taken as $\pm 1.5 \%$ and for optical constants ( $\mathrm{n}$ and $\mathrm{k}$ ) as $\pm 2.5 \%$.

\section{Results and discussion}

Fig. 1 presents the spectral distribution of transmittance $\mathrm{T}(\lambda)$ and reflectance $\mathrm{R}(\lambda)$ for Si crystals with ( $\left.\begin{array}{lll}1 & 0 & 0\end{array}\right)$ and ( 1111$)$ orientations, in the spectral range of $200 \mathrm{~nm}$ to $2500 \mathrm{~nm}$. It shows that the spectral distribution of transmittance for Si crystals has strong absorption at $\lambda \approx 1000 \mathrm{~nm}$. However, the spectral distribution of reflectance for Si crystals is 
characterized by sharp structures at $\lambda<1000 \mathrm{~nm}$, which are associated with high energy transitions from the valence band to conduction band. At $\lambda>$ $1000 \mathrm{~nm}$, the reflectance decreases with increasing the wavelength.

The transmittance and reflectance values were used to calculate the absorption coefficient $\alpha$ according to the following equation [5-7]:

$\alpha=$

$$
(1 / d) \ln \left\{(1-R)^{2} / 2 T+\left[\left((1-R)^{4} / 4 T^{2}\right)+R^{2}\right]^{1 / 2}\right\}
$$

where the sample thickness is $\mathrm{d}$ in $\mathrm{cm}$. The variation of the absorption coefficient $\alpha$ as a function of incident photon energy h $v$ is shown in Fig. 2.

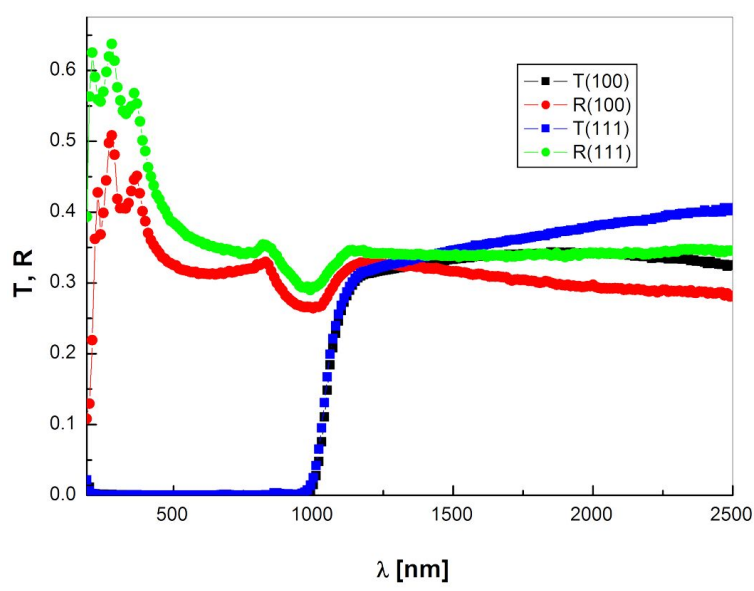

Fig. 1. Spectral distributions of transmittance, $\mathrm{T}$, and reflectance, R, for $\mathrm{Si}$ single crystals.

The inset of Fig. 2 shows the photon energy dependence of extinction coefficient $\mathrm{k}$, where $\mathrm{k}=\alpha \lambda / 4 \pi$. The value of $\alpha$ rises with increasing photon energy for Si crystals with $\left(\begin{array}{lll}1 & 0 & 0\end{array}\right)$ and ( $\left.\begin{array}{lll}1 & 1 & 1\end{array}\right)$ orientations.

The dependence of absorption coefficient on photon energy was studied to get information about the optical energy gap. The relation between $\alpha$ and $\mathrm{h} v$ is given as $[8,9]$ :

$$
(\alpha h v)=Y\left(h v-E_{g}\right)^{p}
$$

where $\mathrm{Y}$ is a constant, $\mathrm{E}_{g}$ is the optical energy gap and $\mathrm{p}$ is an index that describes the optical absorption process. The parameter $\mathrm{p}$ has the values of 2 ,

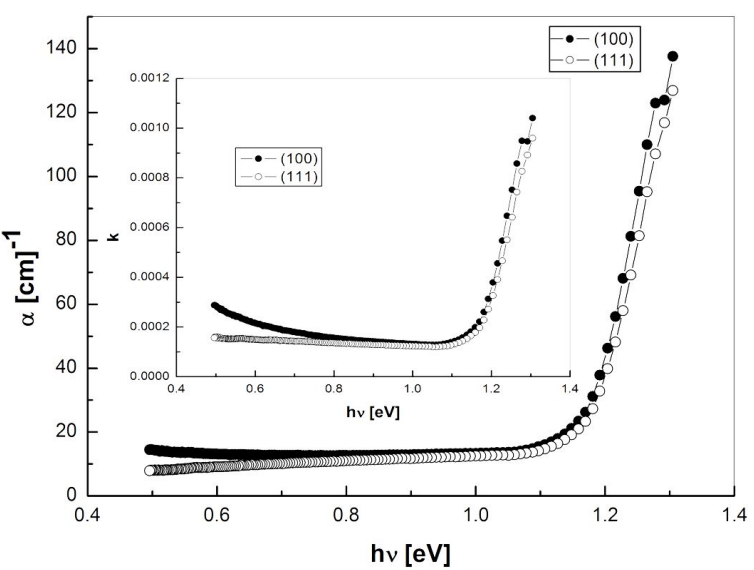

Fig. 2. Variation of absorption coefficient $\alpha$, against the photon energy hv; the inset figure shows the dependence of extinction coefficient $\mathrm{k}$, on the photon energy for Si single crystal.

$3,1 / 2$ and $3 / 2$ for indirect allowed, indirect forbidden, direct allowed and direct forbidden transitions, respectively. The plot of $(\alpha \mathrm{h} v)^{1 / 2}$ against $h v$ gives the best fit linear relation in the absorption region near the absorption edge, as shown in Fig. 3. The figure satisfies the equation [8]:

$$
(\alpha h v)^{1 / 2}=Y\left(h v-E_{g}^{i n d} \pm E_{p h}\right)
$$

where $\mathrm{E}_{\mathrm{ph}}$ is the phonon energy. It shows that the absorption takes place through the indirect allowed transition. The value of $\mathrm{E}_{\mathrm{g}}$ is about $1.1 \pm 0.025 \mathrm{eV}$ for Si crystals. Thus, it is independent of the orientation. The phonon energy associated with the indirect transition was found to be $\sim 21.12 \mathrm{meV}$ and

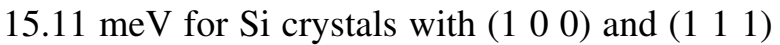
orientations, respectively.

The spectral distribution of the refractive index (n) was determined from the reflectance $R$ and the extinction coefficient $(\mathrm{k})$ values at the same wavelength using the following relation [10]:

$$
n=[(1+R) /(1-R)]+\left[\left(4 R /(1-R)^{2}\right)-k^{2}\right]^{1 / 2}
$$

The refractive index $n$ is shown in Fig. 4 as a function of $h v$. The spectral behavior of $n$ shows an anomalous dispersion at photon energy $>1.1 \mathrm{eV}$, which is characterized by the existence of several peaks at different energies, which are tabulated in Table 1 for different orientations. These peaks may 

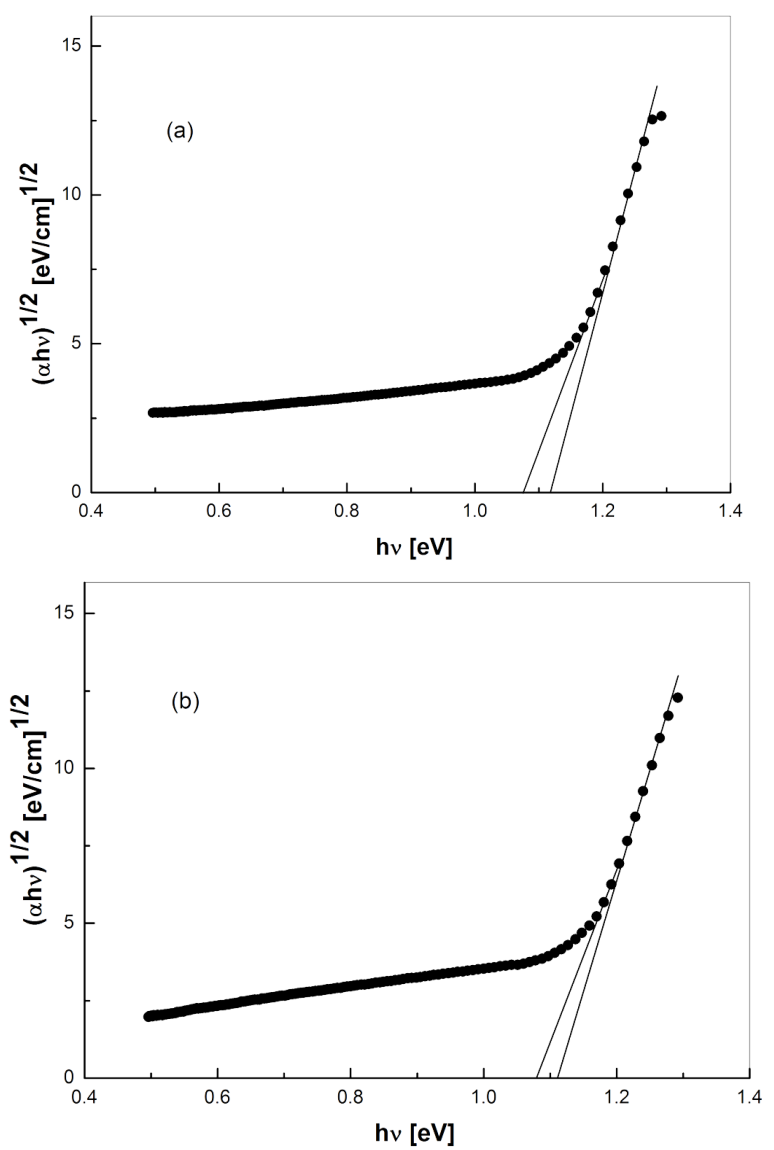

Fig. 3. Plot of $(\alpha h v)^{1 / 2}$ against the photon energy $h v$ : (a) for $\mathrm{Si}\left(\begin{array}{lll}1 & 0 & 0\end{array}\right)$, and (b) for $\mathrm{Si}\left(\begin{array}{lll}1 & 1 & 1\end{array}\right)$ single crystals.

be attributed to the band structure of the material [12]. Also, the refractive index shows a normal dispersion behavior at photon energy $<1.1 \mathrm{eV}$.

Table 1. Interband transition corresponding to peak positions in the refractive index spectrum for $\mathrm{Si}$ single crystals with different orientations.

\begin{tabular}{lccc}
\hline \multirow{2}{*}{ Orientation } & \multicolumn{3}{c}{ Transitions [11] } \\
\cline { 2 - 4 } & $\Gamma_{25^{\prime}}-\Gamma_{15}$ & $\mathrm{X}_{4}-\mathrm{X}_{1 \mathrm{c}}$ & $\mathrm{L}_{3^{\prime}}-\mathrm{L}_{3}$ \\
\hline \hline$\left(\begin{array}{lll}1 & 0 & 0\end{array}\right)$ & 3.35 & 4.43 & 5.31 \\
$\left(\begin{array}{lll}1 & 1 & 1\end{array}\right)$ & 3.44 & 4.43 & 5.91 \\
Si $\left[\begin{array}{llll}1 & 1\end{array}\right]$ & 3.43 & 4.4 & 5.5 \\
\hline
\end{tabular}

The energy dependence of the refractive index $\mathrm{n}$ in the normal dispersion region can be analyzed according to the single oscillator model proposed by Wemple-DiDomenico [13, 14]. The refractive

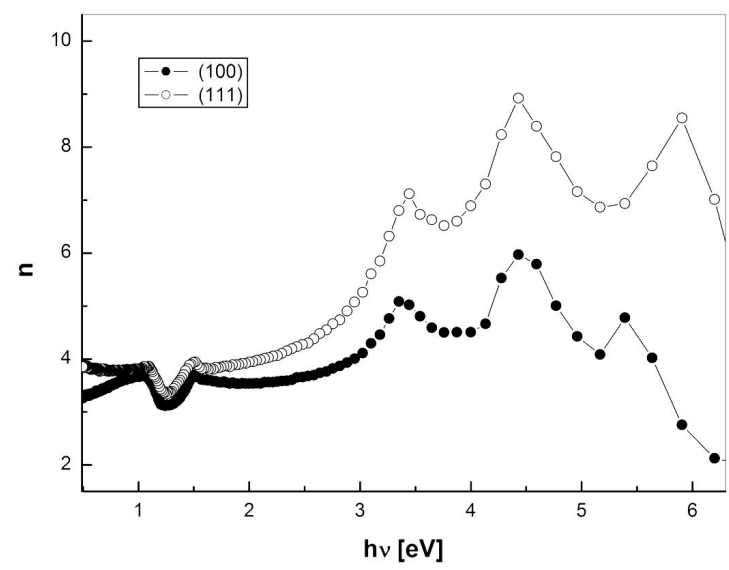

Fig. 4. The photon energy dependence of the refractive index $\mathrm{n}$ for Si single crystals.

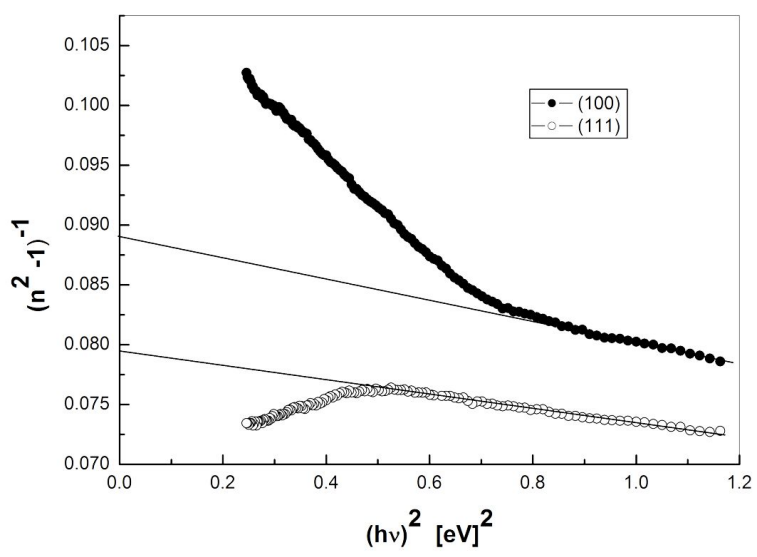

Fig. 5. Plot of $\left(n^{2}-1\right)^{-1}$ against $(h v)^{2}$ for Si single crystals.

index is related to photon energy according to the relation:

$$
\left(n^{2}-1\right)^{-1}=\left(E_{o} / E_{d}\right)-\left(1 / E_{o} E_{d}\right) \cdot(h v)^{2}
$$

where $E_{o}$ is the oscillator energy and $E_{d}$ is the dispersion energy. The dispersion parameters were determined by plotting $\left(\mathrm{n}^{2}-1\right)^{-1}$ against $(\mathrm{h} v)^{2}$ as shown in Fig. 5 for Si crystals of different orientations. The refractive index $\mathrm{n}(0)$ at zero photon energy, which is defined by the high frequency dielectric constant $\epsilon_{\infty}$, was deduced from the dispersion relationship by extension the linear portion like in Fig. 5. The values of $E_{o}, E_{d}$ and $\epsilon_{\infty}$ were calculated and recorded in Table 2. 
Table 2. Optical parameters of Si crystals with different orientations.

\begin{tabular}{lcccccc}
\hline Orientation & $\mathrm{E}_{\mathrm{ph}}[\mathrm{meV}]$ & $\mathrm{E}_{\mathrm{o}}[\mathrm{eV}]$ & $\mathrm{E}_{\mathrm{d}}[\mathrm{eV}]$ & $\epsilon_{\infty}$ & $\epsilon_{\mathrm{L}}$ & $\mathrm{N} / \mathrm{m}^{*}\left[\mathrm{~cm}^{-3} \cdot \mathrm{g}^{-1}\right]$ \\
\hline \hline$(100)$ & 21.12 & 3.33 & 37.48 & 12.24 & 12.9 & $4.64 \times 10^{47}$ \\
$(111)$ & 15.11 & 3.66 & 46.13 & 13.59 & 14.61 & $1.53 \times 10^{47}$ \\
\hline
\end{tabular}

An important contribution of the WempleDiDomenico model is interpretation of dispersion energy in terms of the chemical bonding. The dispersion energy $E_{d}$ is related to other physical parameters of material by the following empirical relationship [12, 15]:

$$
E_{d}=\beta \cdot N_{c} \cdot Z_{a} \cdot N_{e}
$$

where $\mathrm{N}_{\mathrm{e}}$ is the effective number of valence electrons per anion, $\mathrm{N}_{\mathrm{c}}$ is the effective coordination number of the cation nearest neighbor to the anion and $Z_{a}$ is the formal chemical valence of the anion. The parameter $\beta$ has two values; the ionic value $\beta_{\mathrm{i}}=0.26 \pm 0.03 \mathrm{eV}$ and covalent value $\beta_{\mathrm{c}}=$ $0.37 \pm 0.04 \mathrm{eV}$. $\beta$ was calculated as 0.30 and 0.36 for Si crystals with ( $\left.\begin{array}{lll}1 & 0 & 0\end{array}\right)$ and $\left(\begin{array}{lll}1 & 1 & 1\end{array}\right)$ orientations, respectively. It indicates that the material has a covalent structure.

The refractive index $\mathrm{n}$ is expressed as a function of the wavelength $\lambda$ by the following relation [16]:

$$
n^{2}=\varepsilon_{L}-\left(e^{2} / \pi c^{2}\right) \cdot\left(N / m^{*}\right) \cdot \lambda^{2}
$$

where $\mathrm{N} / \mathrm{m}^{*}$ is the ratio of carrier concentration to its effective mass and $\epsilon_{\mathrm{L}}$ is the lattice dielectric constant. The plot of $\mathrm{n}^{2}$ versus $\lambda^{2}$ is linear at longer wavelength as illustrates Fig. 6. This behavior is due to the contribution of free carriers absorption and lattice vibration modes of dispersion [12]. The extrapolation of the linear part of the plot $\mathrm{n}^{2}$ versus $\lambda^{2}$ to $\lambda^{2}=0$ yields the value of the lattice dielectric constant $\epsilon_{\mathrm{L}}$, which varies from 12.9 to 14.61 depending on the crystal orientation. The $\mathrm{N} / \mathrm{m}^{*}$ ratio was determined using the slope of the linear part of the plots in Fig. 6 and tabulated in Table 2.

Another parameter can be deduced from the refractive index behavior according to the ClausiusMossotti model. This model introduces physically the electronic polarizability $\left(\alpha_{\mathrm{e}}\right)$ by reducing

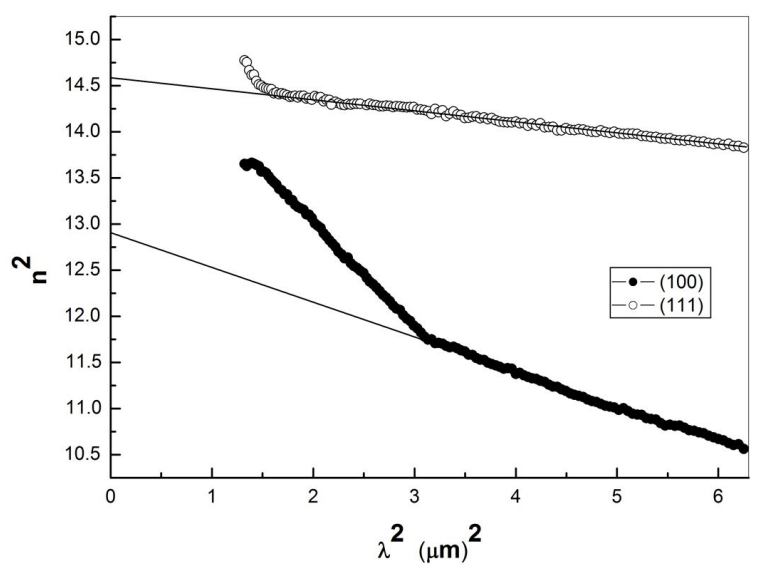

Fig. 6. Plot of $n^{2}$ versus $\lambda^{2}$ for Si single crystals.

the model to the well-known Lorentz-Lorenz formula [12]. The Lorentz-Lorenz relation is written as $[12,17]$ :

$$
\left(n^{2}-1\right) /\left(n^{2}+2\right)=\left(N_{A} \rho / 3 M \varepsilon_{o}\right) \cdot \alpha_{e}
$$

where $\mathrm{N}_{\mathrm{A}}$ is the Avogadro number, $\rho$ is the mass density, $M$ is the molecular weight of the material and $\epsilon_{\mathrm{o}}$ is the permittivity of the free space. The photon energy dependence of $\left(n^{2}-1\right) /\left(n^{2}+2\right)$ for Si crystals with $\left(\begin{array}{lll}1 & 0 & 0\end{array}\right)$ and $\left(\begin{array}{lll}1 & 1 & 1\end{array}\right)$ orientations is shown in Fig. 7. From the extrapolation and equation 8 , the electronic polarizability $\alpha_{\mathrm{e}}$ was determined to be $3.84 \times 10^{-40} \mathrm{Fm}^{2}$ and $4.38 \times 10^{-40} \mathrm{Fm}^{2}$ at $\mathrm{h} v=0$ for $\mathrm{Si}\left(\begin{array}{lll}1 & 0 & 0\end{array}\right)$ and $\mathrm{Si}\left(\begin{array}{lll}1 & 1 & 1\end{array}\right)$ orientations, respectively.

On the other hand, the real and imaginary parts of dielectric constant of Si crystals were also estimated by knowing the values of the refractive index and extinction coefficient. The real and imaginary parts of complex dielectric constant are expressed as [18]:

$$
\varepsilon_{1}=n^{2}-k^{2}, \quad \varepsilon_{2}=2 n k
$$

where $\epsilon_{1}$ is the real part and $\epsilon_{2}$ is the imaginary part of complex dielectric constant. 


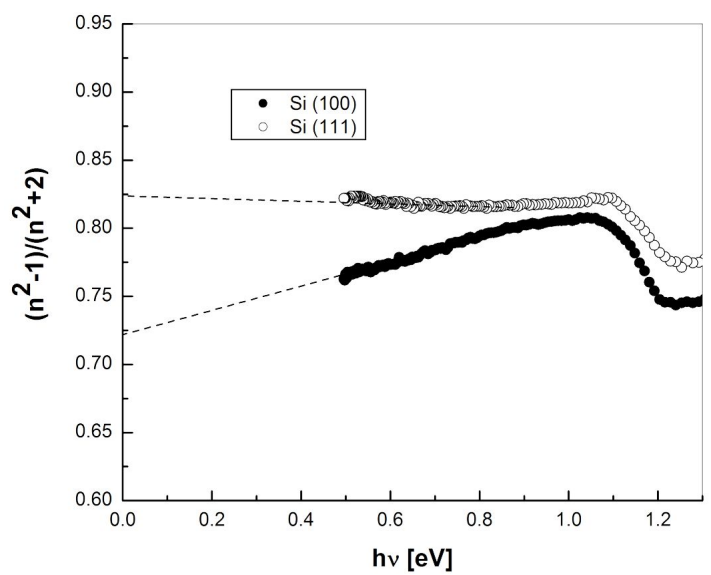

Fig. 7. Variation of $\left(n^{2}-1\right) /\left(n^{2}+2\right)$ with $h v$ for $\mathrm{Si}$ single crystals.

The dependences of $\epsilon_{1}$ and $\epsilon_{2}$ on photon energy are shown in Fig. 8 and Fig. 9.

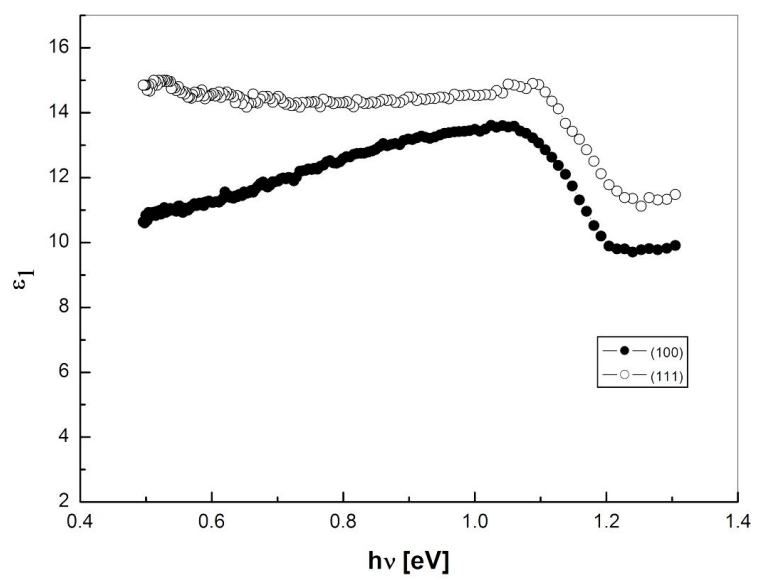

Fig. 8. Variation of $\epsilon_{1}$ as a function of $\mathrm{h} v$ for Si single crystals.

\section{Conclusions}

The optical properties of $\mathrm{Si}$ single crystals with two orientations $\left(\begin{array}{lll}1 & 0 & 0\end{array}\right)$ and $\left(\begin{array}{lll}1 & 1 & 1\end{array}\right)$ were studied using transmittance and reflectane measurements in the spectral range of $200 \mathrm{~nm}$ to $2500 \mathrm{~nm}$. The optical absorption data revealed the existence of indirect allowed transition band with energy gap of $1.1 \pm 0.025 \mathrm{eV}$. On the basis of Wemple and DiDomenico single oscillator model,

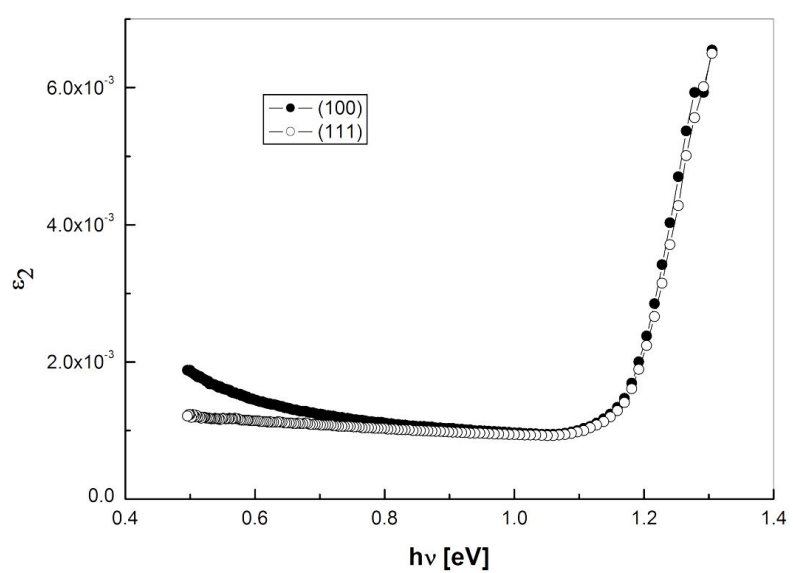

Fig. 9. Variation of $\epsilon_{2}$ as a function of $h v$ for Si single crystals.

the parameters such as of $E_{0}, E_{d}, \epsilon_{\infty}, \epsilon_{L}$ and $\beta$ factor were evalauted. The electronic polarizability $\alpha_{\mathrm{e}}$ was determined for the different orientations and it was found to be $3.84 \times 10^{-40} \mathrm{Fm}^{2}$ and $4.38 \times 10^{-40} \mathrm{Fm}^{2}$ at $\mathrm{h} v=0$ for $\mathrm{Si}\left(\begin{array}{lll}1 & 0 & 0\end{array}\right)$ and $\mathrm{Si}$ (1 111 1) orientations, respectively. The dependence of the real and imaginary parts of dielectric constant on photon energy was also investigated.

\section{References}

[1] Al-NaimeE K.A., Indian J. Sci. Technol., 3 (2010), 308.

[2] Sokolov V.I., Shelykh A.I., Tech. Phys. Lett.+, 34 (2008), 196.

[3] Puzder A., Williamson A.J., Grossman J.C., Galli G., J. Chem. Phys., 117 (2002), 6721.

[4] Pawlak B.J., Gregorkiewicz T., AmmerlaAn C.A.J., Takkenberg W., Tichelaar F.D., AlkeMAdE P.F.A., Phys. Rev. B, 64 (2001), 115308.

[5] El-Nahass M.M., ATta A.A., El-Sayed H.E.A., El-Zaidia E.F.M., Appl. Surf. Sci., 254 (2008), 2458.

[6] El-Nahass M.M., Youssef S.B., Ali H.A.M., J. Optoelectron. Adv. M., 13 (1) (2011), 76.

[7] Giulio Di M., MicocCi G., Rella R., SicilIAno P., TEWre A., Phys. Status Solidi A, 136 (1993), KlOl.

[8] Pankove J.I., Optical Processes on semiconductors, Prentice-Hall, New York, 1971.

[9] TAUC J., Amorphous and liquid semiconductors, Plenum Press, New York, 1974.

[10] Moss T.S., Optical Properties in semiconductor, Butter Worths, London, 1959.

[11] Wakaki M., Kudo K., Shibuya T., Physical Properties and Data of Optical Materials, CRC, New York, 2007. 
[12] El-Gohary Z., El-Nahass M.M., Soliman H., EL-KADY Y.L., J. Mater. Sci. Technol., 19 (2003), 77.

[13] Wemple S.H., Didomenico M., Phys. Rev. B, 3 (1971), 1338.

[14] Wemple S.H., Phys. Rev. B, 7 (1973), 3767.

[15] Sharma P., Vashistha M., Jain I.P., Chalcogenide Lett., 2 (2005), 115.

[16] Andre J.J., Simon J., Molecular Semiconductors, Spring-Verlag, Berlin, 1985.
[17] GonZalez-Leal J.M., VlceK Mir., PrietoAlcon R., Stronski A., Wagner T., Marquez E., J. Non-Cryst. Solids, 326 - 327 (2003), 146.

[18] Caglar M., Ilican S., Caglar Y., Phys. Macedonica, 56 (2006), 49.

Received 2017-08-06

Accepted 2018-12-21 